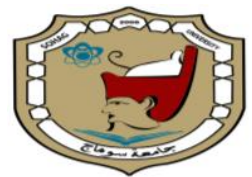

Sohag University
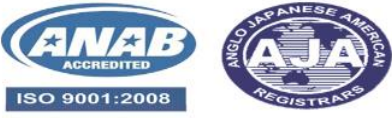

Sohag Medical Journal

Faculty of Medicine

\title{
Can platelet count alone predict bleeding in patients with thrombocytopenia?
}

\author{
Marwa Zanaty Elsayed ${ }^{1}$, Hamdy S Mohammed ${ }^{1}$, Eman H Salama ${ }^{2}$, \\ Lotfy Hamed Abo-Dahab'.
}

1- internal medicine department, Faculty of Medicine, Sohag University

2- clinical pathology department, Faculty of Medicine, Sohag University

\begin{abstract}
:
The number of platelets is used to assess thrombopoiesis; it varies greatly between individuals, although it is relatively steady in healthy people, with a modest drop with age. The severity of thrombocytopenia is a considerable factor in determining bleeding risk. However, while some patients with platelet numbers less than $10,000 \mathrm{~L}^{-}$ ${ }^{1}$ have a little bleeding, others with platelet numbers above $50,000 \mathrm{~L}^{-1}$ have large hemorrhages, indicating that platelet count alone may not necessarily predict the occurrence of bleeding in patients with a marked decrease in platelet number. Platelet function testing in thrombocytopenia may be useful as a diagnostic tool and as guidance to treatment for thrombocytopenic patients. Severe thrombocytopenia is a major risk factor for hemorrhage, but platelet function and bleeding risk at very low platelet counts are poorly understood due to the limitations of platelet function testing at very low platelet count. Using flow cytometry is generally independent of platelet number and hence possible in blood from individuals with thrombocytopenia.

Keywords: Platelet count, platelet function, thrombocytopenia, bleeding.
\end{abstract}

\section{Introduction:}

Thrombocytopenia is the most prevalent reason for hematologic consultation in medical practice, which is also one of the first most life-threatening illnesses ${ }^{(1)}$.

Platelets are important in the early stages of clot formation because they adhere to injured blood vessels and donate their membrane phospholipids to activate coagulation factors $(2)$.

\section{Degrees of thrombocytopenia}

Thrombocytopenia is described as a platelet number that is less than the normal cutoff value, which in adults is $150,000 /$ microL. Mild thrombocytopenia (platelet count 100,000 to $150,000 /$ microL), moderate thrombocytepenia (platelet count 50,000 to 99,000/ microL), and severe thrombocyteepenia (platelet count 50,000/ mic-
roL). These numbers should be assessed according to the etiology, lower or higher levels may be acceptable in some circumstances. For example, in the setting of immune thrombocytopenia [ITP], we consider a platelet count of 30,000 as severe thrombocytopenia (3). The hazard of spontaneous mucocutaneous bleeding and potentially fatal spontaneous cerebral hemorrhage or gastrointestinal bleeding rises rapidly below $10 \times 10^{9} / \mathrm{L}^{(4)}$.

\section{Causes of thrombocytopenia}

It may be due to a decrease in platelet synthesis (hypo-productive) or acceleration in platelet consumption/destructtion (hyper-destructive) thrombocytepenia ${ }^{(5)}$.

Hematological malignancies (leukemia, myeloma, myelodysplasia, and my- 
elofibrosis), cytotoxic drugs or radiotherapy, infections (human immuneodeficiency virus (HIV), Cytomegalovirus (CMV), hepatitis $\mathrm{B}$, and $\mathrm{C}$ ), aplastic and megaloblastic anemia, and generalized bone marrow failures are all examples of hypo-productive thrombocytopenia ${ }^{(6)}$.

Idiopathic/primary autoimmune (ITP), Secondary (systemic lupus erythematosus, chronic lymphocytic leukemia, lymphoma), Infections (HIV, hepatitis $\mathrm{B}$, and C, malaria), Drug-induced (rifampicin, penicillin, sulphonamides, $\mathrm{He}-$ parin, quinine), and disseminated intravascular hemolysis (DIC) are all examples of hyper-destructive thrombocytopenia $^{(1)}$

\section{Platelet dysfunction may be a cause of bleeding in thrombocytopenic pat- ients}

Platelet bleeding disorders are a diverse group in terms of frequency and bleeding severity; they are characterized by qualitative/function and/or quantitative/number platelet defects; However, when moderate $\left(50-100^{*} 10^{9}\right.$ platelets/L) or even mild thrombocytopenia (>100-150*109 platelets/L) is combined with hereditary or acquired defect in platelet function, bleeding owing to substantial hemostatic problems, such as operations and injury, is very common ${ }^{(7,8)}$

Although serious bleeding is more frequent when platelet numbers are below $10,000 \mathrm{~L}^{-1}$, considerable hemorrhage can occur at higher platelet counts as well, the vast majority of individuals with even severe thrombocytopenia do not complain of spontaneous bleeding because of the presence of another cause of bleeding other than thrombocytopenia. ${ }^{(9,10)}$.

As a result, while severe thrombocytepenia cause bleeding, it is not adequate. In a study of patients with hematologic malignancies who were having stem cell transplantation or chemotherapy, bleeding of World Health Organization grade 2 or above occurred at rates of $25 \%$ at platelet counts of $5000 \mathrm{~L}^{-1}$ and $17 \%$ at platelet levels of $\left(20-80000 \mathrm{~L}^{-1}\right)^{(10)}$.

Although 75 percent of intracranial hemorrhages (ICHs) resulted at platelet counts $10000 \mathrm{~L}^{-1}, 10 \%$ of ICHs occured at platelet counts $>20000 \mathrm{~L}^{-1}(9)$ external events such as trauma or infection, as well as differences in platelet function, may lead to variations in bleeding risk among patients with nearly equivalent degrees of thrombocytopenia. Furthermore, because the types of bleeding differ, a better understanding of platelet function in thrombocytepenia could be a significant aspect that determines the risk of bleeding and, if so, could lead to better clinical management ${ }^{(11)}$.

Whereas bleeding is common in hematological cancers with bone marrow failure ${ }^{(12)}$. bleeding is assumed to be fewer in thrombocytopenic conditions of increased platelet turnover, such as thrombocytopenic purpura (TTP), heparin-induced thrombocytopenia, and immune thrombocytopenia (ITP). In these conditions, the platelet number decreases due to depletion of platelet due to increased coagulation processes; and transfusion of platelet may lead to the occurrence of thrombosis and fatality ${ }^{(13)}$.

marked thrombocytopenia is a substantial risk factor for bleeding, however, assessment of the relation between the possibility of occurrence of bleeding and the presence of defects in platelet function in thrombocytopenic patients remain unclear due to the difficulties in the platelet function measurements in severe thrombocytopenia ${ }^{(8)}$.

The use of Flow cytometry in the assessment of platelet function: - 
Flow cytometry represents the standard technique to quantify receptors and distinct platelet activation markers on the surface of platelets in citrated whole blood for the evaluation of quantitative receptor defects and increased platelet activation status in vivo, including the shedding of surface membrane receptors. The in vitro assessment of platelet reactivity in response to a variety of agonists, especially in low amounts of whole blood, demonstrates further strengths (14).

Platelet function assay relies on singlecell analysis using flow cytometry is generally independent of platelet number and hence possible in blood from individuals with thrombocytopenia. $\mathrm{H}$ owever, based on a new study, when platelet number $10^{*} 10^{9} / \mathrm{mL}$ may affect in vitro platelet activation tests because of platelet count-related decreased release of ADP, which serves as an important amplifier of platelet activation ${ }^{(15)}$.

\section{References:}

1. Jumaa Khaleel K, Anwer Ahmed AJKJoM. Platelet indices and their relations to platelet count in hypoproductive and hyper-destructive Thrombocytopenia. 2014;7(2):1952-8.

2. Sang Y, Roest M, de Laat B, de Groot PG, Huskens DJBR. Interplay between platelets and coagulation. 2021;46:100733.

3. George J, Arnold D. Immune thrombocytopenia (ITP) in adults: Secondline and subsequent therapiesUpToDate. UpToDate; 2020.

4. Vinholt PJJCC, Medicine L. The role of platelets in bleeding in patients with thrombocytopenia and hematological disease. 2019;57(12):1808-17.

5. Islam S, Islam $M$, Ahmed M, Aziz $M$, Begum MJHTIJ. Role of mean platelet volume (MPV), platelet distribution width (PDW) and platelet large cell ratio (P-LCR) value in the diagnosis of immune thrombocytopenic purpura. 2016;2(2):1-5.
6. Zulfania Z, Hayat $\mathrm{H}$, Mahmood R, Bukhari AA, Ihtesham Y, Rasool UJPJOP. COMPARISON OF PLATELET INDICES IN HYPOPRODUCTIVE AND HYPERDESTRUCTIVE THROMBOCYTOPENIA. 2021;17(2):3-6.

7. Gresele P, Kleiman NS, Lopez JA, Page CP. Platelets in thrombotic and nonthrombotic disorders: Pathophysiology, pharmacology and therapeutics: An update: Springer; 2017.

8. Jurk K, Shiravand YJJocm. Platelet Phenotyping and Function Testing in Thrombocytopenia. 2021;10(5):1114.

9. Psaila B, Petrovic A, Page LK, Menell J, Schonholz M, Bussel JBJB. Intracranial hemorrhage (ICH) in children with immune thrombocytopenia (ITP): study of 40 cases. 2009;114(23):4777-83.

10. Slichter SJ, Kaufman RM, Assmann SF, McCullough J, Triulzi DJ, Strauss $\mathrm{RG}$, et al. Dose of prophylactic platelet transfusions and prevention of hemorrhage. 2010;362(7):600-13.

11. Bolton-Maggs PHJp, health C. Management of immune thrombocytopenic purpura. 2007;17(8):305-10.

12. Heddle NM, Cook RJ, Sigouin C, Slichter SJ, Murphy M, Rebulla P, et al. A descriptive analysis of international transfusion practice and bleeding outcomes in patients with acute leukemia. 2006;46(6):903-11.

13. Goel R, Ness PM, Takemoto CM, Krishnamurti L, King KE, Tobian AAJB, The Journal of the American Society of Hematology. Platelet transfusions in platelet consumptive disorders are associated with arterial thrombosis and in-hospital mortality. 2015;125(9):1470-6.

14.Jurk KJH. Analysis of platelet function and dysfunction. 2015;35(01):60-72.

15.Boknäs N, Macwan AS, Södergren AL, Ramström SJR, thrombosis pi, haemostasis. Platelet function testing at low platelet counts: When can you trust your analysis? 2019;3(2):285-90. 